\title{
Simulation of Transients in the end Synchronous Generatorin Matlab Package
}

\author{
Konstantin S. Fediy, \\ Sergey A. Vstovskiy and Nikolay E. Poloshkov* \\ Siberian Federal University \\ 79 Svobodny, Krasnoyarsk, 660041, Russia
}

Received 21.02.2017, received in revised form 18.05.2017, accepted 26.06.2017

Mathematical modeling in the energy sector, even the simplest and most widely used devices, leading to huge cost savings and improved product quality. The more complex the proposed facility, the usually more important than the role of modeling in learning and creating. The most widely used is modeling in mechanics and physics, electrical engineering, radio engineering and electronics, signal processing and communications technology.

The paper solved the problem of the study of transients in the end the synchronous generator with permanent magnets in the MatLab package. The processes occurring in synchronous machines during transient conditions, such as a sudden short-circuit and sudden load changes. Processes occurring in synchronous machines during transient conditions, such as sudden short circuits or abrupt change of load, are very complex, causing considerable difficulties in their exact quantitative calculation. However, the behavior of the synchronous machine under these conditions is of great practical importance, since transients can cause damage to the machine and, therefore, significant losses related to interruption of power supply facilities, receiving power from the generator, or the termination of work electric drives with synchronous motors.

The peculiarity of the end of the mathematical model of the synchronous generator with magnetoelectric excitation is the replacement of the permanent magnets on the "fictitious" field winding with the included in it a source of current, the magnetomotive force equal to "fictitious" coercive force.

Keywords: end generator, synchronous machine, transients, a mathematical model, the library SimPowerSystems, Machines Measurement Demux.

Citation: Fediy K.S., Vstovskiy S.A., Poloshkov N.E. Simulation of transients in the end synchronous generatorin matlab package, J. Sib. Fed. Univ. Eng. technol., 2017, 10(5), 691-698. DOI: 10.17516/1999-494X-2017-10-5-691-698.

(C) Siberian Federal University. All rights reserved

* Corresponding author E-mail address: fediy_k@mail.ru,wstw2@mail.ru 


\title{
Моделирование переходных процессов
}

\section{в торцевом синхронном генераторе в пакете MATLAB}

\author{
К.С. Федий, С.А. Встовский, Н.Е. Полошков \\ Сибирский федеральный университет \\ Россия, 660041, Красноярск, пр. Свободный, 79
}

\begin{abstract}
Математическое моделирование в энергетике даже самых простых и широко распространенных устройств ведет к огромной экономии средств и улучшению качества изделий. Чем сложнее проектируемыйобъект, тем, какправило, важнее рольмоделирования в его изучении и создании. Самое широкое применение моделирование находит в механике и физике, электротехнике, радиотехнике и электронике, в технике обработки сигналов и коммуникачий.

В статье решена задача исследования переходных проиессов в ториевом синхронном генераторе с постоянными магнитами в пакете MatLab. Исследованы процессы, возникающие в синхронных машинах при переходных режимах, таких как внезапное короткое замыкание и резкое изменение нагрузки. Процессы, возникающие в синхронных машинах при переходных режимах, например при внезапном коротком замыкании или резком изменении нагрузки, весьма сложны, что вызывает значительные трудности при их точном количественном расчете. Однако поведение синхронной машины при указанных режсмах имеет очень большое практическое значение, так как переходные процессы могут вызвать повреждение машины, а следовательно, и значительные убытки, связанные с перерывом энергоснабжения объектов, получающих питание от генератора, или прекращением работы электроприводов с синхронными двигателями.

Особенностью математической модели ториевого синхронного генератора с магнитоэлектрическим возбуждениемявляетсязамена постоянныхмагнитов на «фиктивную» обмотку возбуждения с включенным в неё источником тока, равным магнитодвижущей силе «фиктивной» коэриитивной сильь.
\end{abstract}

Ключевые слова: ториевой генератор, синхронная машина, переходные проиессы, математическая модель, библиотека SimPowerSystems, Machines Measurement Deтих.

Математическое моделирование в энергетике даже самых простых и широко распространенных устройств ведет к огромной экономии средств и улучшению качества изделий. Чем сложнее проектируемый объект, тем, как правило, важнее роль моделирования в его изучении и создании. Самое широкое применение моделирование находит в механике и физике, электротехнике, радиотехнике и электронике, в технике обработки сигналов и коммуникаций. В свою очередь, успехи в этом направлении способствуют созданию аппаратных и программных средств математического моделирования, поэтому нередко для экономии времени и средств исследования процессов, протекающих внутри системы, приходится заменять компьютерным моделированием. Математическое моделирование позволяет понять физическую и математическую сущности моделируемых явлений и обосновать оптимальные подходы к проектированию самых различных изделий.

Представление любой электрической машины в каком-либо пакете программ сводится к составлению уравнений, описывающих эту машину, и принятию некоторых допущений, вводимых для упрощения расчетов. Расчет и составление данных уравнения машины можно вести двумя методами:

- во вращающейся системе координат; 
- в системе координат, жестко связанной с ротором.

Для удобства расчета выберем вариант с неподвижной системой координат относительно ротора машины.

Таким образом, выражая переменные, описывающие характер протекания процессов и характеризующие свойства машины (потокосцепление, ток и напряжения в каждой фазе трехфазной системы) через новые коэффициенты, привязанные к какой-либо системе координат, избавляются от периодических коэффициентов дифференциальных уравнений синхронной машины.

Особенностью математической модели торцевого синхронного генератора с магнитоэлектрическим возбуждением является замена постоянных магнитов на «фиктивную» обмотку возбуждения с включенным в неё источником тока $I_{B}^{\prime}=c o n s t$, равным МДС «фиктивной» коэрцитивной силы $F_{\text {мф }}=H_{c \phi} \cdot l_{\text {м }}$.

Библиотека блоков SimPowerSystems выступает одной из множества дополнительных библиотек Simulink пакета MatLab, ориентированных на моделирование конкретных устройств. Несомненным достоинством SimPowerSystems признано то, что сложные электротехнические системы можно моделировать, сочетая методы имитационного и структурного моделирования. Например, силовую часть полупроводникового преобразователя электрической энергии можно выполнить с использованием имитационных блоков SimPowerSystems, а систему управления - с помощью обычных блоков Simulink, отражающих лишь алгоритм ее работы, а не ее электрическую схему. Такой подход в отличие от пакетов схемотехнического моделирования позволяет значительно упростить всю модель, а значит, повысить ее работоспособность и скорость работы. Кроме того, в модели с использованием блоков SimPowerSystems (далее SPS-модели) можно применять блоки и остальных библиотек Simulink, а также функции самого MatLab, что дает практически неограниченные возможности для моделирования электротехнических систем.

На рис. 1 представлена схема модели генератора с постоянными магнитами, реализованная при помощи пакета MatLab в Simulink. Особенность схемы заключается в том, что она

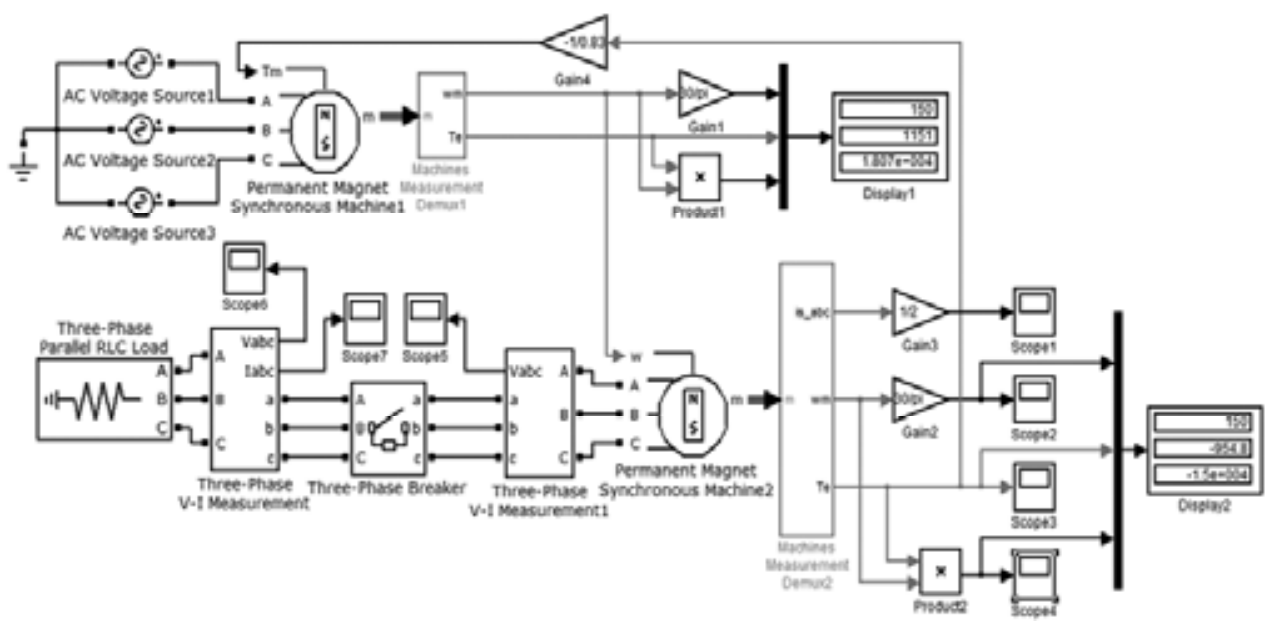

Рис. 1. Модель генератор-двигатель 
позволяет посмотреть переходные процессы сразу как в двигательном, так и в генераторном режиме.

При реализации модели возникает проблема задания частоты вращения привода генератора из-за отсутствия в библиотеке Simulink стандартных двигателей с малой частотой вращения ротора. Эта проблема решается использованием двух одинаковых синхронных машин с постоянными магнитами характеристики, которых рассчитаны с помощью разработанной математической модели. Выходная мощность двигателя, необходимая для вращения генератора, рассчитывается по формуле

$$
P_{\partial}=\frac{P_{2}}{\eta_{2}}
$$

где $P_{2}$ - мощность генератора, кВт; $\eta_{2}-$ КПД генератора, о.е.

Так, например, для генератора мощностью 15 кВт требуется приводной двигатель мощностью $P_{\partial}=15 / 0,83=18,07$ кВт.

Основной элемент модели - блок Permanent Magnet Synchronous Machine, являющийся моделью синхронной машины с постоянными магнитами из электротехнической библиотеки SimPowerSystems (рис. 2).

Порты модели А, В и С - выводы статорной обмотки машины. Входной порт Тт служит для задания механического момента. На выходном порту m формируется векторный сигнал, состоящий из 10 элементов:

- 1-3: токи обмотки статора $-i_{s a}, i_{s b}, i_{s c}$;

- 4-5: проекции токов статора на оси $d$ и $d-i_{d}, i_{q}$;

- 6-7: проекции напряжений статора на оси $d$ и $d-U_{d}, U_{q}$;

- 8: угловая частота вращения ротора $-\omega_{r}$;

- 9: угол поворота ротора - $\theta$;

- 10: электромагнитный момент $-T_{e}$.

Для удобства извлечения переменных машины из выходного вектора измеряемых переменных в библиотеке SimPowerSystems предусмотрен блок Machines Measurement Demux.

Электрическая часть модели машины описывается системой уравнений, связанных с ротором [1]:

$$
\frac{d}{d t} \cdot i_{d}=\frac{1}{L_{d}} \cdot U_{d}-\frac{R}{L_{d}} \cdot i_{d}+\frac{L_{q}}{L_{d}} \cdot p \cdot \omega_{r} \cdot i_{q}
$$

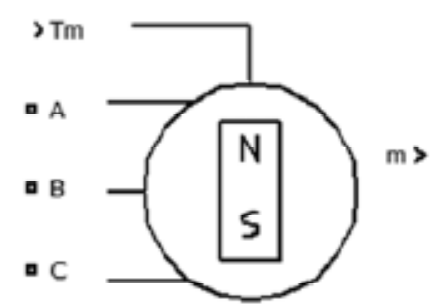

Рис. 2. Блок Permanent Magnet Synchronous Machine 


$$
\begin{aligned}
& \frac{d}{d t} \cdot i_{q}=\frac{1}{L_{q}} \cdot U_{q}-\frac{R}{L_{q}} \cdot i_{q}+\frac{L_{d}}{L_{q}} \cdot p \cdot \omega_{r} \cdot i_{d}-\frac{\lambda \cdot p \cdot \omega_{r}}{L_{q}}, \\
& T_{e}=1,5 \cdot p \cdot\left(\lambda \cdot i_{d}+\left(L_{d}-L_{q}\right) \cdot i_{d} \cdot i_{q} .\right.
\end{aligned}
$$

Все параметры ротора и его переменные приведены к статору. В системе уравнений приняты следующие обозначения:

- $L_{d}, L_{q}$ - индуктивности статора по осям $d$ и $d$;

- $R$ - активное сопротивление обмотки статора;

- $\lambda$ - магнитный поток постоянных магнитов, сцепленный с обмоткой статора.

Механическая часть модели описывается уравнениями:

$$
\begin{aligned}
& \frac{d}{d t} \cdot \omega_{r}=\frac{1}{J} \cdot\left(T_{e}-F \cdot \omega_{r}-T_{m}\right), \\
& \frac{d}{d t} \cdot \theta=\omega_{r},
\end{aligned}
$$

где $J$ - суммарный момент инерции ротора и нагрузки; $F$ - коэффициент трения; $\theta$ - угол положения ротора; $T_{m}$ - момент сопротивления.

Процессы, возникающие в синхронных машинах при переходных режимах, например при внезапном коротком замыкании или резком изменении нагрузки, весьма сложны, что вызывает значительные трудности при их точном количественном расчете [2]. Однако поведение синхронной машины при указанных режимах имеет очень большое практическое значение, так как переходные процессы могут вызвать повреждение машины, а следовательно, и значительные убытки, связанные с перерывом энергоснабжения объектов, получающих питание от генератора, или прекращением работы электроприводов с синхронными двигателями [3].

На рис. 3 и 4 представлены переходные процессы, происходящие в обмотке статора ТСГ на постоянных магнитах.

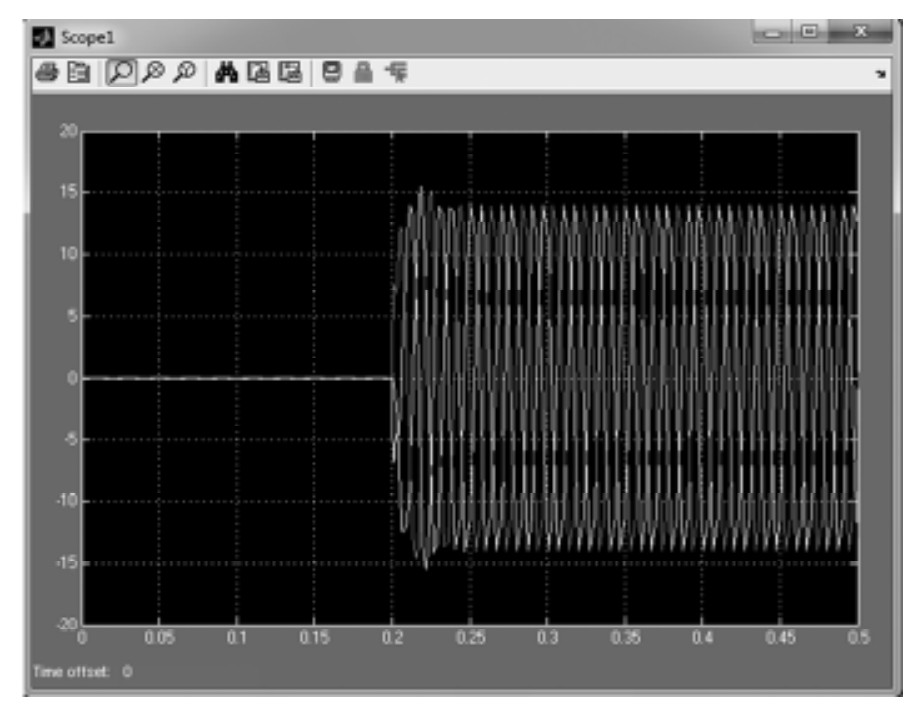

Рис. 3. График токов обмотки статора генератора

$$
-695-
$$




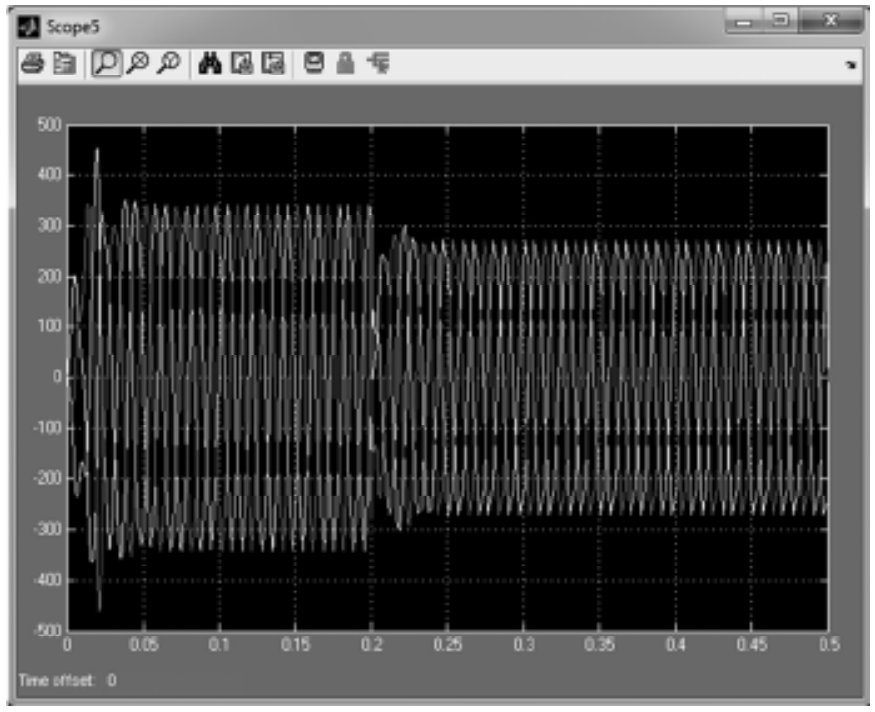

Рис. 4. График напряжений обмотки статора генератора

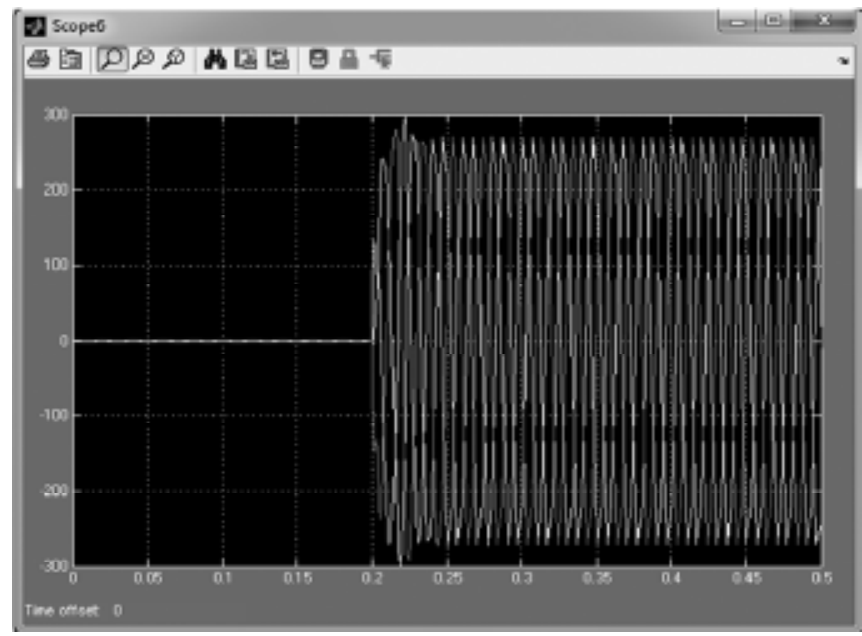

Рис. 5. График напряжений на номинальной нагрузке

В момент времени 0 с происходит пуск генератора, при этом появляются скачки напряжения. В период времени с 0,05 до 0,2 с генератор работает в установившемся режиме холостого хода. В момент времени 0,2 с происходит замыкание ключа и к генератору подводится активная нагрузка, при этом появляются скачки тока (рис. 3) и падение напряжения в обмотке статора (рис. 4). С момента времени 0,25 с генератор работает уже в установившемся режиме работы на номинальную нагрузку (рис. 5).

На рис. 6-8 видно, что в начальный момент короткого замыкания (0,2 c) машина испытывает сильный удар за счет резко возросших токов статорной обмотки и кинетической энергии ротора. В результате этого возникает тормозящий момент, имеющий характер кратковременного импульса, во время которого скорость машины падает до 60 об/мин, поэтому необходимо 


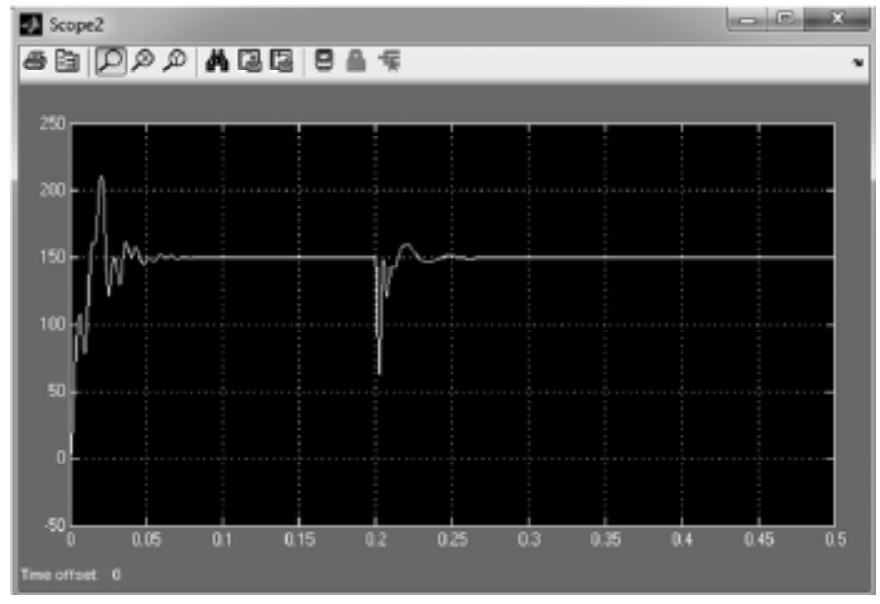

Рис. 6. График частоты вращения ротора

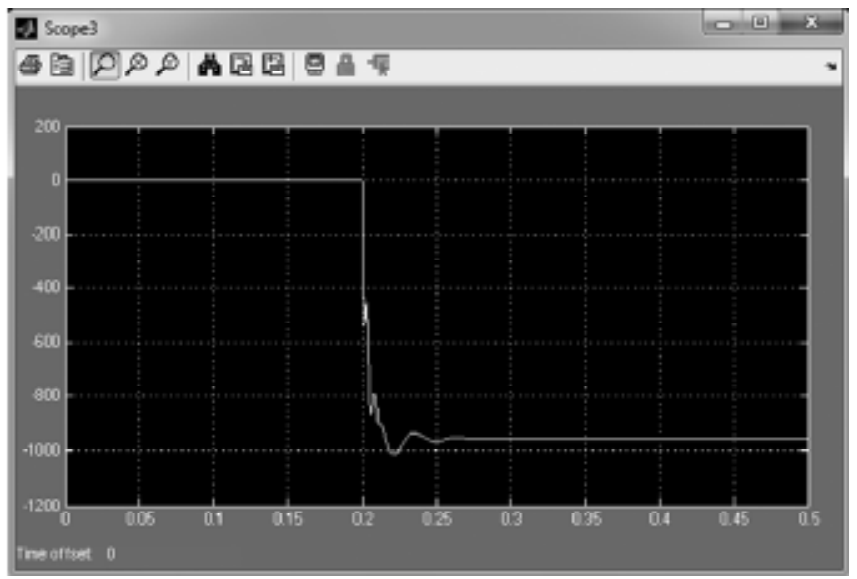

Рис. 7. График электромагнитного момента

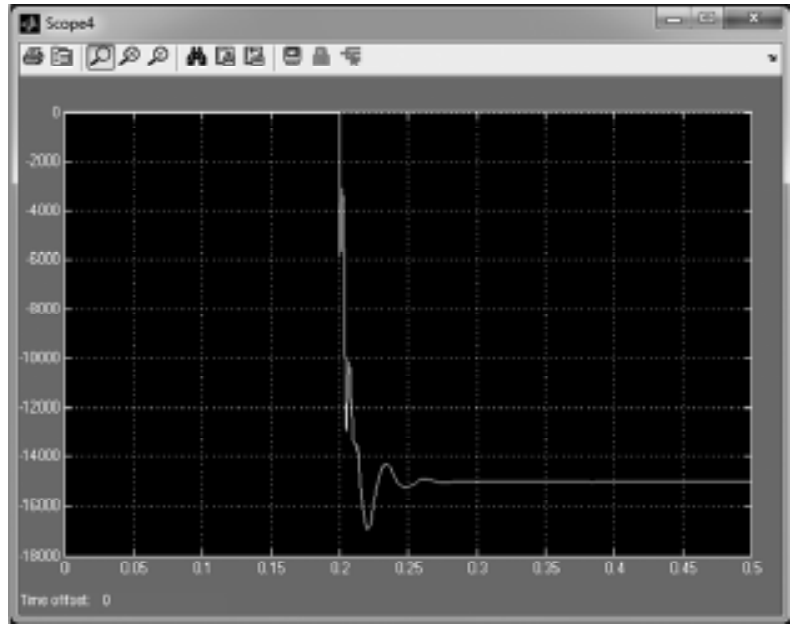

Рис. 8. График выходной мощности 
уделять большое внимание обеспечению механической прочности машины, прежде всего ее активной части.

Графическое значение номинального тока обмотки статора составляет 13.9 А, что очень близко к расчетному значению номинального тока 14.2 А. Расхождение результатов моделирования и результатов аналитического расчета незначительно, что позволяет предположить правильность построения имитационной модели ТСГ на постоянных магнитах и задания параметров моделирования.

Исследование переходных процессов при внезапном трехфазном коротком замыкании рассматривались для исследования величин токов, возникающих при коротком замыкании, и определения динамических сил, возникающих вследствие взаимодействия токов короткого замыкания и магнитных полей.

Определение величин токов короткого замыкания и их изменения во времени в первую очередь особо важно для конструкторов электрических сетей и распределительных устройств, так как динамическая нагрузка устройств определяется максимальным значением тока короткого замыкания, а изменение токов короткого замыкания во времени имеет решающее значение при выборе выключателей.

Проведенные исследования показали, что вид графиков при внезапном трехфазном коротком замыкании практически идентичен полученным графикам переходных процессов при сбросе и набросе нагрузки, что связано с тем, что постоянные магниты имеют малую магнитную проницаемость $\left(\mu \approx 1,5-4 \mu_{0}\right)$.

\section{Список литературы}

[1] Осин И.Л., Шакарян Ю.Г. Электрические машины: Синхронные машины: ред. И.П. Копылов. М.: Высш. шк., 1990. 304 с. [Osin I.L., Shakaryan Yu.G. Electric cars: Synchronous machines: Ed. I.P. Kopylov. M.: Higher. Rk, 1990. 304 p. (in Russian)]

[2] Важнов А.И. Основы теории переходных проиессов синхронной машины. Ленинград, 1960. [Vazhnov A.I. Basic theory of transients of synchronous machine. Leningrad, 1960. (in Russian)]

[3] Сипайлов Г.А., Кононенко Е.В., Хорьков К.А. Электрические машины (специальный курс): М.: Высш. Шк., 1987. 287 с. [Sipajlo G.A., Kononenko E.V., Ferrets K.A. Elektricheskie mashiny (special course): M.: Higher. Rk, 1987. 287 p. (in Russian)] 